\title{
A New Strategy for Efficient Decentralized Network Control
}

\author{
Evariste Logota $^{1,2}$, Augusto Neto ${ }^{1,3}$, Susana Sargento ${ }^{1,2}$, \\ ${ }^{1}$ Institute of Telecommunications, Aveiro, Portugal \\ ${ }^{2}$ University of Aveiro, Portugal \\ ${ }^{3}$ Federal University of Goiás, Goiânia, Brazil \\ logota@av.it.pt, augusto@inf.ufg.br, susana@ua.pt
}

Abstract-The limited scope of Internet architecture forced network providers implementing centralized mechanisms (QoS, group communication, mobility, etc) to support attractive value added sessions. Besides ease to manage, performance aspects of such mechanisms, mainly in terms of scalability, robustness and availability, are shortcoming. In this sense, decentralization is promising, since complex networking operations may be provided throughout the nodes, increasing the network performance. However, current distributed solutions implement their own strategies for decentralization (e.g. synchronization, resilience, etc.), which contributes to increase Internet's complexity and damage overall performance. This paper introduces a cost-effective strategy for scalable decentralization of control operations in current Internet, called Self-Organizing Multiple Edge Nodes (SOMEN) mechanism. SOMEN enables multiple distributed network Control Decision Points -CDPs(e.g. network borders) to jointly exploit control data inside a network with low signaling load. The effectiveness of SOMEN was proved through analytical modeling and simulation, which demonstrated significant minimization of control overhead.

Keywords- distributed network control, self-management, performance optimization, network protocol, Quality of Service.

\section{INTRODUCTION}

With the overwhelming trend of social, economic and technological merging, the Internet is envisioned to become the core infrastructure with high transport capacity so as to connect different access networks for content exchanges. Hence, key features and capabilities taking into account reliability, scalability and robustness are required to support attractive value added sessions. The list of such sessions includes mission critical applications (location based systems, geo processing/monitoring, health-care, security, etc.) for a large number of users anywhere and anytime (ubiquitous) with acceptable quality at reasonable cost. For this purpose, the network infrastructures must provide cost-effective solutions to operators, being efficiently used without unnecessary control overheads.

As the original Internet architecture was not specified with solutions to support different services, such as QoS and multicast, solutions on top of correct layers (overlay) were designed to minimize this issue. Overlay solutions are mainly deployed in a centralized way, such as for QoS (TISPAN [1]) and group communications (Application Layer Multicast [2]). Besides not requiring deeper changes in a system, overlay can damage overall network performance by placing excessive control overhead in the network (signaling, state, processing and energy overload). Recent studies [3]-[4] claim that decentralized approaches fit better in large-scale environments than centralized solutions. In one hand, decentralization allows the support of simultaneous operations at entities throughout a network, achieving better performance with less resources (e.g., bandwidth, CPU and memory) consumption. On another hand, decentralization requires the synchronization of control information of multiple decision points to avoid wrong and incompatible decisions. Therefore, decentralization must be correctly designed to prevent network performance degradations. Moreover, inexistence of standards for decentralization force each distributed system to deploy its own strategies. As a result, Internet's complexity increases even more by the addition of new protocols and mechanisms.

This paper introduces the Self-Organizing Multiple Edge Nodes (SOMEN), a generic-purpose mechanism to minimize the impact in network performance of decentralized networking control mechanisms in current and future Internet scenarios. The main idea consists in enabling multiple distributed Control Decision Points - CDPs (e.g., network borders) to jointly exploit control data inside a network in support for key control sub-systems (e.g. traffic engineering, over-reservation, end-to-end transport control, link capacity planning, etc.), in a way that allows improving system overall performance in a flexible and cost-effective manner, i.e. keeping low control loads. The cost-effectiveness of SOMEN was demonstrated through analytical modeling while its support for over-reservation control in decentralized networks, was proved by implementing as a use case of over-provisioning centric QoS-aware multicast solution, called Multi-user Aggregated Resource Allocation (MARA) [5]. This use case was chosen due to its complexity and inability to work in multiple distributed CDPs' environments. For this purpose, MARA was deployed with SOMEN decentralized operations, and simulations have been carried out to examine its performance. Results show that it is possible to provide efficient decentralization with significantly low control signaling overheads.

The paper is organized as follows. Section II presents the related works, while section III describes SOMEN approach. In section IV SOMEN is evaluated. Finally, section V draws the conclusion and future work.

\section{RELATED WORK}

Today, there is a rapidly increasing dependence of citizen and business on IT technologies, making central controllers more and more bottlenecked while communication networks are subject to frequent failures. Hence, decentralization supporting redundant connectivity and multihoming with 
automated control capabilities appeared promising for scalability, robustness, availability, [3]-[6]-[7]. However, synchronization control signaling overheads pose serious problems in decentralization (e.g. P2P networks [8], Ad hoc networks [9], etc.) where exceeding signaling consumes too much resources in terms of bandwidth, memory, CPU and energy.

In literature, it is widely researched that knowledge of network topology and links state information, mainly the available resources [10] on bottleneck links and their location on paths [11], are of paramount importance for researchers and Internet Service Providers (ISPs) to improve system overall performance. Hence, current designs mostly enable nodes in the network to maintain knowledge of underlying network topology in terms of nodes, interfaces, capacities, list of onpath outgoing interfaces, etc [5]-[12]-[11]. However, most designs are currently centralized or hierarchical.

In recent QoS control proposals it is claimed that over provisioning is promising for scalability. However, overreservation control strongly requires available bandwidth on paths' bottleneck links without probing paths, so as to overcome the performance degradations of per-flow reservation mechanisms. However, such requirement cannot be fully met by current measurement algorithms, especially in decentralized networks as cross-traffic loads make links' state parameters highly unpredictable [13]-[14]-[15]. In [5], the Multi-user Aggregated Resource Allocation (MARA) introduced a dynamic bandwidth over-reservation scheme, which distinguished itself among value-added state of the art. However, MARA's algorithm was evaluated in a very simple architecture, with a single control point (one ingress router). Therefore, MARA fails in complex scenarios such as in decentralized networks being envisioned.

Motivated by the hereinabove challenges and requirements, we believe that further research for efficient decentralization control is necessary to support future complex scenarios. It is required to envision proposals that minimize undue control signaling overheads, which may outweigh benefits or damage network performance (e.g. synchronization overheads, measurement overheads, QoS control signaling overheads, etc.). Hence, we investigate a decentralized approach that is able to make use of correlation between paths and provide an efficient solution for network control.

\section{SOMEN MECHANISM}

This section aims to introduce SOMEN approach with its functionalities proposed in support for a decentralization control.

\section{A. SOMEN Overview}

In addition to the traditional network topological information, SOMEN enables CDPs to efficiently maintain communication paths correlation patterns inside a network cluster. The main idea is to jointly exploit correlation patterns and traffic load dynamics in various correlated paths to infer cross traffic and links key state information with low signaling overheads. Thus, CDPs are enabled to efficiently cooperate, exchange control information, and quickly adapt to changes in network states.

The operations are implemented by two control agents: SOMEN-Edge agent (SOMEN-E) and SOMEN-Core agent
(SOMEN-C). SOMEN-E is a statefull agent deployed by CDPs and implements the main SOMEN functions. SOMEN-C is a lightweight-state agent deployed by control Decision Enforcement Points (DEPs), and simply implements appropriate functions to properly interpret and process SOMEN control signaling messages for decision enforcement (e.g. resource control, message forwarding, etc.) and report of resilience events (e.g. link/node failures) to CDPs. Thus, SOMEN pushes control complexity to CDPs and leaves DEPs simple for further scalability purposes.

As in the SOMEN-aware network illustrated in Fig. 1, the border routers of each cluster (clusters A, B, and C) are configured as CDPs by deploying SOMEN-E, while interior routers are configured as DEPs as they deploy SOMEN-C. We suggest that each cluster deploy its own control model in a way that assures end-to-end paths over heterogeneous transport control models, where clusters can inter-connect based on Service Level Agreement (SLA) or specific contracts defined by their providers.

The next section describes in detail the main SOMEN functionalities.

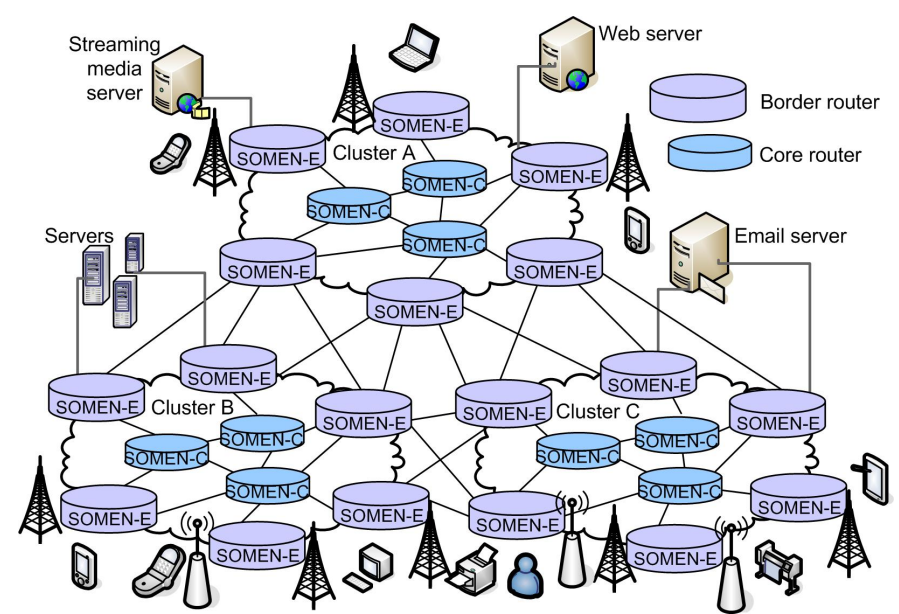

Figure 1. An illustration of SOMEN agents throughout a network

\section{B. SOMEN Functionalities}

The SOMEN functions include: (a) Dynamic Filtering Functions (DFF); (b) Dynamic Threshold Synchronization Functions (DTSF); and (c) Network Context Information Base (NetCIB) Resilience Functions (NRF).

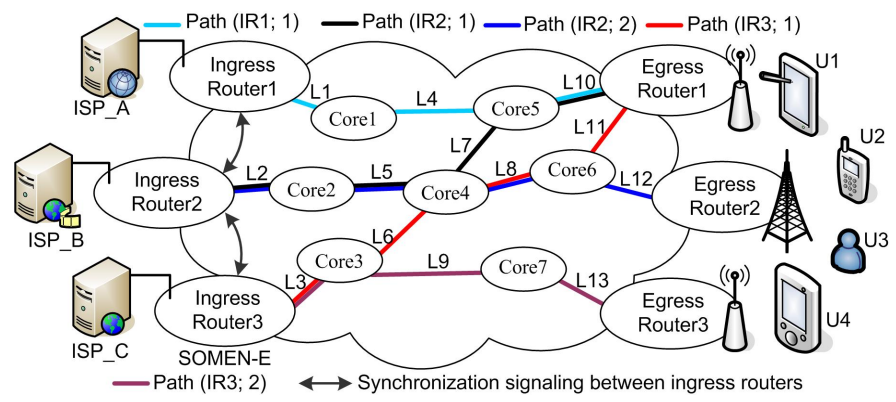

Figure 2. SOMEN-aware single network domain

To provide a better understanding of SOMEN approach, we start our descriptions based on the decentralized network cluster of Fig. 2. Hence, for the sake of simplicity, the ingress 
routers 1, 2, and 3 are configured as SOMEN-aware CDPs hosting SOMEN-E agent, while other routers, such as the egress routers 1, 2, 3 and the 7 core routers, are configured as SOMEN-aware DEPs hosting SOMEN-C agent. Subscribers (U1, U2, U3, or U4) are expected to receive services with acceptable quality from their Providers (ISP_A, ISP B or ISP C) at reasonable cost. In the rest of the paper, CDP notation is used relating to an ingress router hosting SOMEN-E agent.

\section{1) Dynamic Filtering Functions}

The DFF implemented by SOMEN-E agent assures every CDP within a cluster to dynamically learn and maintain communication paths' correlation patterns (in addition to the traditional network topological information), and links resource conditions in a database called Network Information Base (NetCIB). In addition, it is expected that each CDP quickly adapts to changes with low control loads. For this purpose, at system initialization where each CDP builds its own NetCIB database, we assume that network administrator first has a strong influence on the booting up of the network. Thus, at system bootstrap, when no session is running yet, each CDP deploys appropriate signaling protocol and creates many edgeto-edge paths. As briefly illustrated in Fig. 2, Ingress router1 creates the path (IR1, 1), ingress router2 creates the paths (IR2, 1) and (IR2, 2), while ingress router3 creates the paths (IR3, 1) and $(I R 3,2)$. In this paper, a path represented by (IRx, ID) can be read as path ID originated at ingress router $x$. In order to maximize system throughput and resource utilization efficiency, a CDP is allowed to create multiple paths between itself and any other border router within a domain. In our specific use case, MARA, in its base form it is created by a flooding-based method (details can be found in [5], which is out of SOMEN scope) for paths creation at system bootstrap.

As CDPs have created paths independently, they use SOMEN to exchange with one another appropriate information about the paths created. The information they exchange includes the paths' IDs, the used bandwidth in each class of service $(\mathrm{CoS})$ on each path, and the list of outgoing interfaces that lie on paths. Thus, upon receiving the advertisement messages, also denoted as synchronization messages in this paper, every CDP builds the so-called NetCIB database mainly composed of PATHS, CORRELATIONS, TOPOLOGY, and LISTS tables (e.g. Table 1 to Table 4).

Table 1 PATHS of ingress router 3

\begin{tabular}{|c|c|c|c|c|c|c|c|c|}
\hline \multirow{2}{*}{$\begin{array}{c}\text { Ingress } \\
\text { router IDs }\end{array}$} & \multirow{2}{*}{$\begin{array}{c}\text { Paths } \\
\text { IDs }\end{array}$} & \multirow{2}{*}{$\begin{array}{c}\text { Multicast } \\
\text { Channels }\end{array}$} & \multirow{2}{*}{$\begin{array}{c}\text { Egress } \\
\text { Router IDs }\end{array}$} & \multirow{2}{*}{$\begin{array}{c}\text { List of on-path } \\
\text { nodes/links }\end{array}$} & \multicolumn{2}{|c|}{ Used Bw (Mbps } & \multicolumn{2}{|c|}{$\begin{array}{c}\text { VOPRs } \\
\text { (Mbps) }\end{array}$} \\
\cline { 6 - 10 } & & & & & $\mathrm{AF}$ & $\mathrm{EF}$ & $\mathrm{AF}$ & $\mathrm{EF}$ \\
\hline 3 & 1 & $(3,1)$ & 1 & L3; L6; L8; 111 & 0 & 0 & 1 & 1.5 \\
\hline 3 & 2 & $(3,2)$ & 3 & L3; L9; L13 & 0 & 0 & 1 & 1.5 \\
\hline
\end{tabular}

Table 2 CORRELATIONS of ingress router 3

\begin{tabular}{|c|c|c|}
\hline Links IDs & Ingress routers IDs & List of path/tree IDs \\
\hline L3 & 3 & $1 ; 2$ \\
\hline L6 & 3 & 1 \\
\hline L8 & 3 & 1 \\
\hline L8 & 2 & 2 \\
\hline L9 & 3 & 2 \\
\hline L11 & 3 & 1 \\
\hline L13 & 3 & 2 \\
\hline
\end{tabular}

Table 3 TOPOLOGY of ingress router3

\begin{tabular}{|c|c|c|c|c|c|c|c|c|c|c|}
\hline \multirow{2}{*}{$\begin{array}{c}\text { Links } \\
\text { IDs }\end{array}$} & \multirow{2}{*}{$\begin{array}{c}\text { Links } \\
\text { Capacity } \\
(\mathrm{Mbps})\end{array}$} & \multicolumn{2}{|c|}{$\begin{array}{c}\text { Reserved } \\
\text { (Mbps) }\end{array}$} & \multicolumn{2}{|c|}{ Used (Mbps) } & \multicolumn{2}{|c|}{$\begin{array}{c}\text { Available } \\
\text { (Mbps) }\end{array}$} & \multirow{2}{*}{$\begin{array}{l}\text { Sharing } \\
\text { Factor } \omega\end{array}$} & \multicolumn{2}{|c|}{$\begin{array}{c}\text { Available/ } \omega \\
\text { (Mbps) }\end{array}$} \\
\hline & & $\mathrm{AF}$ & EF & $\mathrm{AF}$ & $\mathrm{EF}$ & $\mathrm{AF}$ & $\mathrm{EF}$ & & $\mathrm{AF}$ & $\mathrm{EF}$ \\
\hline L3 & 10 & 2 & 3 & 0 & 0 & 2 & 3 & 2 & 1 & 1.5 \\
\hline L6 & 10 & 2 & 3 & 0 & 0 & 2 & 3 & 1 & 2 & 3 \\
\hline L8 & 10 & 2 & 3 & 0 & 0 & 2 & 3 & 2 & 1 & 1.5 \\
\hline L9 & 10 & 2 & 3 & 0 & 0 & 2 & 3 & 1 & 2 & 3 \\
\hline L11 & 10 & 2 & 3 & 0 & 0 & 2 & 3 & 1 & 2 & 3 \\
\hline L13 & 10 & 2 & 3 & 0 & 0 & 2 & 3 & 1 & 2 & 3 \\
\hline
\end{tabular}

Table 4 LISTS of ingress router3

\begin{tabular}{|c|c|c|}
\hline Ingress routers IDs & Paths/trees IDs & List of advertisement receivers \\
\hline 3 & 1 & 2 \\
\hline 3 & 2 & NULL \\
\hline
\end{tabular}

To ease understanding on the process used by each CDP to build its NetCIB, we use ingress router3 of Fig. 2 as an example. In this context, ingress router 3 first creates its PATHS table as shown in Table 1, and stores the paths originated from itself, the own paths, such as (IR3, 1) and (IR3, 2) (in the rest of this paper, the links composing own paths are called own links). Hence, as CDPs located at ingress routers merge traffics into own paths while traffic behavior is unpredictable, each CDP records the used bandwidth in each $\mathrm{CoS}$ on each path, as in Table 1. This used bandwidth is based on the amount of bandwidth it granted to the related active sessions during QoS negotiation. Thus, traffic conditioning is operational for reshaping or policing out-of-profile flows to control (in average) the traffic load injected in a $\mathrm{CoS}$ on a given path. Hence, whenever a session is admitted, readjusted or terminated in a $\mathrm{CoS}$, the corresponding record of used bandwidth is automatically updated accordingly. Besides, the Virtual Over-PRovisioning (VOPR) of each CoS on the bottleneck link of each path is stored in PATHS table for admission control purposes. Details on VOPRs of CoSs in a path are given later in the paper.

Moreover, ingress router 3 creates its CORRELATIONS table to store paths correlation patterns for own links. This correlation patterns are created such that, for a given link, the patterns relate the IDs of the paths to which it belongs and the corresponding CDPs, as shown in Table 2. Thus, given a tuple (CDP ID; path ID) within a cluster, every CDP is enabled to easily retrieve all own links which correlate with that path and perform relevant processing.

An example is the following: a CDP modifies the used bandwidth $b w$ in a CoS along a path and needs to advertise the new changes of such control data to other correlated CDPs. Hence, it only needs to advertise its own ID, the path ID, the CoS ID and the used bandwidth $b w$, without the list of links that compose the path. Upon receiving the well flagged advertisement message, other CDPs easily use the tuple (one's ID, path ID) to obtain all their correlated links, and properly update related own links and own paths states information according to the CoS ID and $b w$. Thus, both advertisement message size and processing loads are reduced.

In this sense, let $\theta$ be the size of a link ID; $l$ be the number of on-path links, and $\Omega_{a}$ be the number of CDPs to advertise about changes in a path. Thus, advertisement message size can be approximately reduced of $\Delta$ such that:

$$
\Delta=\theta . l . \Omega_{a}
$$


As an example, with $\theta=128$ bit (as in IPv6), $l=5$ on-path/tree links and $\Omega_{a}=50$ CDPs, $\Delta=4$ Kbytes can be reduced every time advertisement should be triggered.

The process of obtaining such correlation patterns for every given link $L_{p}$ is simple. Let's suppose that $n$ paths $\left(P_{1}, P_{2}, \ldots\right.$, $\left.P_{n}\right)$ are created by the CDPs within a cluster. Hence, given an integer $j$, the procedure can be described as:

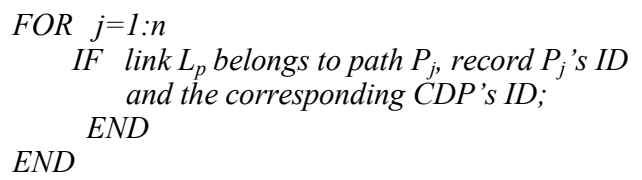

Furthermore, ingress router 3 creates its TOPOLOGY table to maintain own links states parameters as in Table 3, where the Sharing Factor $\omega$ of a link is the number of paths that share the link. The used bandwidth in a $\operatorname{CoS}_{i}$ on a link $L_{p}$ (as in TOPOLOGY table) is the total used bandwidth in $\mathrm{CoS}_{i}$ on $L_{p}$.

The process to obtain such used bandwidth is as follows: let's suppose that $L_{p}$ is shared by $\omega$ paths $\left(P_{1}, P_{2}, \ldots, P_{\omega}\right)$ with respectively used bandwidth $U\left(i, P_{1}\right), U\left(i, P_{2}\right), \ldots, U\left(i, P_{\omega}\right)$ in a $\mathrm{CoS}_{i}$ as recorded in the corresponding CDPs' PATHS tables. Thus, as CDPs exchange their $U\left(i, P_{j}\right)$ bandwidth, and the capacity $C$ of $L_{p}$ as well as the reservation $R\left(i, L_{p}\right)$ for $\mathrm{CoS}_{\mathrm{i}}$ on $L_{p}$ are known, the total accurate used $U\left(i, L_{p}\right)$ and available $A(i$, $L_{p}$ ) bandwidths in a $\operatorname{CoS}_{i}$ on $L_{p}$ are easily obtained by every CDP as:

$$
\begin{aligned}
U\left(i, L_{p}\right) & =\sum_{j=1}^{w} U\left(i, P_{j}\right) \\
A\left(i, L_{p}\right) & =R\left(i, L_{p}\right)-U\left(i, L_{p}\right)
\end{aligned}
$$

Hence, supposing that $k \mathrm{CoSs}$ are available on $L_{p}$, the total used $U\left(L_{p}\right)$ and available $A\left(L_{p}\right)$ bandwidths on $L_{p}$ are given by:

$$
\begin{aligned}
& U\left(L_{p}\right)=\sum_{i=1}^{k} U\left(i, L_{p}\right) \\
& A\left(L_{p}\right)=C-U\left(L_{p}\right)
\end{aligned}
$$

Thus, each CDP populates its NetCIB database with accurate used and available bandwidth on each link together with link's location (link ID) within a network without probing the related paths. Hence, network measurement overhead can be significantly reduced. Moreover, this provides effective support for bandwidth over-provisioning in decentralized networks.

This is also a good approach to provide the knowledge of underlying network state conditions required for key control functions such as traffic engineering, end-to-end transport control, link capacity planning, etc. In particular, the hereinabove mentioned control sub-systems can efficiently exploit NetCIB information, such as available bandwidth on links and links' positions, to perform intelligent load balancing, paths and access points selection, constraint-based routing, avoiding unnecessary congestion within a cluster.

The rest of this sub-section describes how SOMEN DFF allows further minimizing advertisement signaling loads between CDPs.

The ingress router3 exploits PATHS and CORRELATIONS tables to create its LISTS table as in Table 4 , with the main objective of avoiding unnecessary broadcast of advertisement messages (and minimizing synchronization signaling overheads). For this purpose, LISTS table stores the lists of CDPs to advertise about changes in a given path.

The basic rule of this filtering function is: a CDP whose paths do not share any link with the path to be advertised should be filtered out. An example in Table 4 is the following: changes in path (IR3; 1 ) are advertised to ingress router 2 only due to the correlation on links L8 (please see Fig. 2).

To better understand the advantage of this filtering function, let $\Omega_{b}$ be the number of CDPs to advertise without DFF filtering, $\Omega_{c}$ be the number of CDPs to advertise with DFF filtering, and $\Phi$ the size of an advertisement message. Hence, every time an advertisement needs to be sent about changes in a given path $P_{0}$, the control overhead can be approximately reduced of $\Gamma$ as:

$$
\Gamma=\phi \cdot\left(\Omega_{b}-\Omega_{c}\right)
$$

As an example, consider that $\Phi=100 \mathrm{Kbytes}, \Omega_{b}=50 \mathrm{CDPs}$ and $\Omega_{c}=5$ CDPs. Thus, $\Gamma=4.5$ Mbytes of advertisement load is reduced every time an advertisement needs to be sent.

\section{2) Dynamic Threshold Synchronization Functions}

Network links' resource states vary mainly according to events, such as sessions' admission, readjustment or termination inside a network. However, advertisement upon such frequent events in real networks (per-flow advertisement) can place undesired advertisement exchanges overhead between CDPs. Hence, we propose the Dynamic Threshold Synchronization Functions (DTSF) by introducing a dynamically controllable function called Virtual OverPRovisioning (VOPR).

The main idea of VOPR is to enable CDPs to dynamically define and exploit virtual resource for each path based on links that lie on it. Thus, the VOPR of a CoS in a path is represented by the VOPR on the bottleneck link of the path. Hence, every CDP can utilize the virtual resources defined for own paths without issuing advertisement messages, and more importantly, without damaging system performance.

Supposing that a link $L_{p}$ is shared by $\omega$ paths, the VOPR $\gamma_{i}$ of a $\operatorname{CoS}_{i}$ on link $L_{p}$ for a path $P_{0}$ sharing $L_{p}$ is obtained by:

$$
\gamma_{i}=U\left(i, P_{0}\right)+\frac{A\left(i, L_{p}\right)}{\omega}
$$

Where $U\left(i, P_{0}\right)$ is the used bandwidth in $\operatorname{CoS}_{i}$ in $P_{0}$ (obtained from PATHS table), $A\left(i, L_{p}\right)$ is the available bandwidth in $\mathrm{CoS}_{i}$ on link $L_{p}$ as in equation (3).

Hence, when a $\mathrm{CDP}_{j}$ receives a session request $r$ to a $\mathrm{CoS}_{i}$ in a path $P_{0}$ within a cluster, it first checks resource availability in its NetCIB as follows:

$$
\begin{array}{ll}
\text { IF } & \gamma_{i} \geq U\left(i, P_{0}\right)+r \\
& \text { (a) } \gamma_{i} \equiv \text { True; //VOPR is said available } \\
\text { ELSE } & \text { (b) } \gamma_{i} \equiv \text { False; //VOPR is said congested }
\end{array}
$$

Hence, when the VOPR is available ( $\gamma_{i} \equiv$ True ), $\mathrm{CDP}_{j}$ simply processes the request $r$ (e.g. admits, readjusts or terminates session according to instruction carried with $r$ ) and updates its local NetCIB without issuing any advertisement signaling. Thus, per-flow synchronization signaling is avoided without negatively affecting the accuracy of NetCIB, while system performance optimization can be achieved. In this sense, the 
number $\eta$ of connection requests that could be admitted in $\mathrm{CoS}_{i}$ in the paths sharing the bottleneck link of $P_{0}$, without any advertisement signaling until the VOPR gets congested, can be estimated as in the following. Let $P_{0}$ originating from a $\mathrm{CDP}_{j}$ correlate with $y$ other paths originating from $N$ CDPs (including $\mathrm{CDP}_{j}$ ). Among these $y$ paths, suppose that $\omega$ paths share the bottleneck link $L_{p}$ of $P_{0}$. Hence, let $\lambda_{0}$ be the highest rate at which connection requests are assigned to $\mathrm{CoS}_{i}$ in $P_{0}$ to make sure that the VOPR related to $P_{0}$ exhausts first to trigger SOMEN advertisement signaling. Thus, the connection request assignment rate $\lambda_{j}$ to any other paths $P_{j}$ can be expressed as:

$$
\lambda_{j}=\alpha_{j} \cdot \lambda_{0}, \text { with } 0 \leq \alpha_{j}<1
$$

Thus, the total number $\eta$ is approximately computed as:

$$
\eta=\frac{A\left(i, L_{p}\right)}{r . \omega}\left(1+\sum_{j=1}^{\omega-1} \alpha_{j}\right)
$$

However, when the VOPR is congested ( $\gamma_{i} \equiv$ False ), $\mathrm{CDP}_{j}$ advertises other correlated $(N-1)$ CDPs with its ID and the congested path ID in a well flagged advertisement message. Then, upon receiving such advertisement, each of other $(N-1)$ CDPs retrieves own links and own paths, which correlate with the advertised path, and replies to the advertiser with one's ID, the IDs of own correlated paths, and the used bandwidth in each $\mathrm{CoS}$ of each correlated path. Then, when the advertiser receives replies from other CDPs, it computes the total used and available resources in each $\mathrm{CoS}$ on each link composing the congested $P_{0}$ as in equation (2) and (3), respectively. After that, it uses $\sigma$ signaling number (e.g. $\sigma=2 \rightarrow$ reserve+response) for resource configuration along the affected $P_{0}$. After resource control is performed, $\mathrm{CDP}_{j}$ advertises back the correlated CDPs with the available bandwidth in each $\mathrm{CoS}$ on each link on $P_{0}$. Finally, the correlated CDPs send acknowledgement to the advertiser. Thus, all correlated CDPs are allowed to dynamically readjust their VOPRs and quickly adapt to changes. In this sense, the total signaling number $\chi$ (advertisement and QoS reservation signaling) that SOMEN triggers when a VOPR gets congested can be obtained as:

$$
\chi=\mu .(N-1)+\sigma
$$

where $\mu$ is the number of times advertisement signaling messages are exchanged between CDPs every time VOPR gets congested. An example is: $\mu=4 \rightarrow$ first advertisement; replies to advertisement; notification about new resource readjustment results; and acknowledgements.

Hence, SOMEN-aware CDPs are enabled to self-organize and auto-configure themselves while dynamically controlling and efficiently improving network infrastructure utilization in a flexible and cost-effective manner, i.e. with significantly low synchronization signaling loads.

\section{3) NetCIB Resilience Functions}

As the NetCIB is used to improve network overall performance, its reliability and availability must be assured regardless of network dynamic events, such as links/nodes failures which affect path correlations patterns and links' state information.

Keeping this in mind, the NetCIB Resilience Functions (NRF) have been proposed. Hence, SOMEN-C agent contains the list of CDPs IDs which is exploited by DEPs to efficiently report failures to CDPs, allowing the latter to quickly react to such events and properly update their NetCIB accordingly.

An example is the following: upon receiving failure notification about a given link within a cluster, CDPs detect own paths which correlate with it. Then, the traffic flows running in the affected paths are quickly switched to other available paths, to hide such dynamics from affecting sessions, and the NetCIB is updated accordingly. Due to space limitation, the resilience mechanism will not be detailed here.

\section{SOMEN EVALUATION}

The effectiveness of SOMEN control mechanism was evaluated through analytical modeling as well as implementation of MARA over-provisioning algorithm [5] deployed with SOMEN decentralized operations. MARA was chosen to prove SOMEN effective support for over-reservation in multiple distributed CDPs's environments while allowing validating our modeling results. Thus, the simulation results collected from Matlab have been analysed to assess SOMEN decentralization control loads mainly in terms of synchronization signaling overheads minimization.

\section{A. Analytical Model Configuration}

In order to show results from analytical model's perspective, we configured the network control model with appropriate parameters for equation (9) and (10) as summarised in Table 5 where ad, reply and ACK represent respectively advertisement, reply and acknowledgement.

Table 5 Configurations for signaling policies

\begin{tabular}{|c|c|}
\hline Parameters & Values \\
\hline Connection request $r$ & $128 \mathrm{Kbps}$ \\
\hline QoS Reservation Signaling $\sigma$ & $2 \rightarrow$ (reserve + response) \\
\hline$\mu$ & $4 \rightarrow$ (ad; reply; ad; ACK) \\
\hline$\alpha_{i}$ & $8 / 9$ \\
\hline
\end{tabular}

\section{B. Simulation Implementation}

We implemented MARA algorithm [5] in the scenario of Fig. 2, where the CDPs host MARA's edge agent, while the DEPs host the MARA's core agent. For simplicity, we assume that each link (L1, L2,..,L13) in Fig. 2 has the same capacity $C$ and implements 4 CoSs: one Expedited Forwarding (EF), two Assured Forwarding (AF1 and AF2) and one Control Signaling class (CS). As MARA's initial configurations are static and defined by network administrator, we set the Initialization Index to 1/2, the Maximum Reservation Threshold to $C / 3$ and the Committed Reservation Threshold to C/6. Please check [5] for more details on MARA.

Hence, session requests of $128 \mathrm{Kbps}$ each (commonly used in scalable codecs [16]), generated as Poisson processes and set as Constant Bit Rate (CBR) sessions independently by ISP_A, ISP_B and ISP_C, are sent to each CDP (Ingress routers 1, 2, and 3 ). The requests are mapped onto CoSs and trees based on normal distribution function. Then, we consider that no admitted session leaves the network until all the existing 5 paths/trees get congested. In this simulation, a path/tree is considered congested and no future request is assigned to it when a request cannot be admitted after resource readjustment along the path/tree by MARA.

Hence, we performed the assessment with 3 different link capacities $C$ : Switched Ethernet (10Mbps), Switched Fast Ethernet (100Mbps) and Switched FDX Gigabit Ethernet 
(1000Mbps). Then, we collected the performance metrics: number of connection requests received in the network (from all ISPs), number of SOMEN synchronization signaling, number of SOMEN QoS reservation signaling, and SOMEN control signaling (QoS reservation+synchronization signaling) generated by the 3 CDPs (ingress routers) within the network.

\section{Numeric Results}

In Fig. 3, we plotted the total number of connection requests against the total SOMEN signaling number as in equations (9) and (10). The number of signaling messages is considered stable with the total traffic load generated by signaling as the description is not dependent on any specific signaling protocol. Hence, we observed that SOMEN effectively supports several distributed CDPs while it keeps both QoS reservation and synchronization signaling number significantly low as compared to the number of connection requests. This was possible mainly through SOMEN's filtering and threshold based advertisement functions.

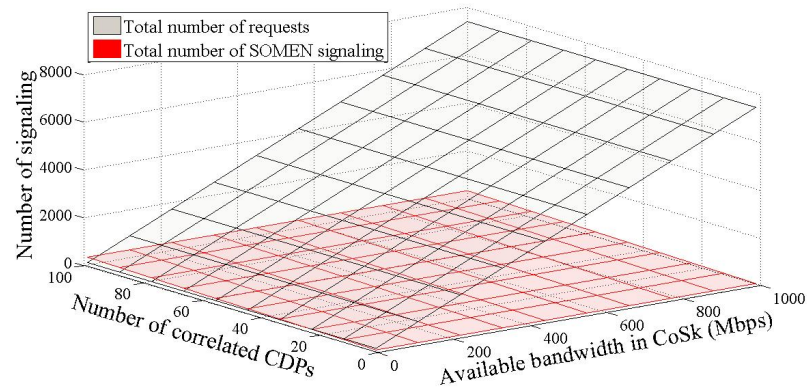

Figure 3. SOMEN modeling results

Fig. 4 shows results with MARA's implementation. As one can see, the total signaling number of SOMEN is very low as compared to session request number. This confirms the modelling results of Fig. 3 in terms of signaling number minimization of SOMEN mechanism. Moreover, it demonstrates SOMEN's effective support for bandwidth overreservation in multiple distributed CDPs' environment.

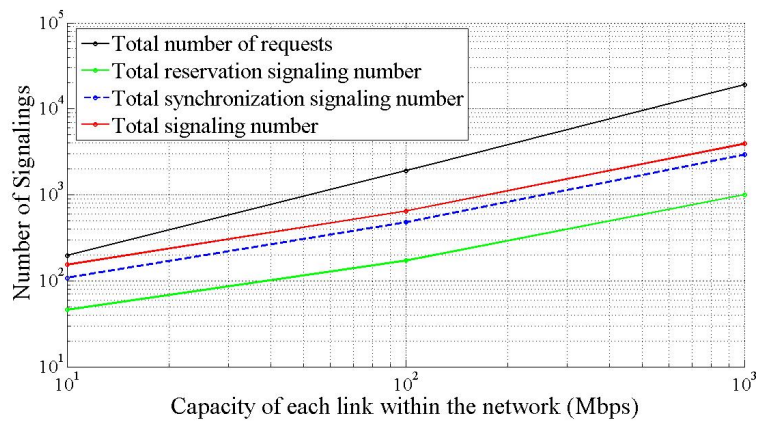

Figure 4. A use case of SOMEN control signaling loads

\section{CONCLUSION \& FUTURE WORK}

This paper proposed SOMEN, a new control mechanism that enables multiple distributed network control decision points to self-control and quickly adapt to changes in network topology. It provides communication paths/trees correlations patterns and the related resource conditions using overreservation in a flexible and cost-effective manner, by keeping low states, processing and signaling overheads. Besides, it pushes control complexity to border routers and leaves interior nodes simple, while presenting potential benefits for network key functions (link capacity planning, end-to-end transport control, paths/trees selection), such that network overall performance can be improved. In the near future, we will further demonstrate the accuracy of the results obtained through Network Simulator-2. Besides, we will focus on SOMEN resilience mechanism with redundant connectivity and multihoming, as well as appropriate signaling protocol to better profit from SOMEN functionalities and relevant advantages.

\section{ACKNOWLEDGMENT}

The work in this paper is supported by the European ICT Context Casting -C-CAST- project (Contract-No. ICT-216462) MuMoMgt Portuguese Foundation of Science and Technology -FCT- project, GEN-CAN IT Project, and the FCT scholarship.

\section{REFERENCES}

[1] ETSI TISPAN - Telecommunications and Internet converged Services and Protocols for Advanced Networking - (www.etsi.org/tispan/).

[2] Suman Banerjee, Bobby Bhattacharjee, Christopher Kommareddy; "Scalable Application Layer Multicast", SIGCOMM'02.

[3] Z. Li, P. Mohapatra, C.-N. Chuah, "Virtual Multi-Homing: On the Feasibility of Combining Overlay Routing with BGP Routing", University of California at Davis Technical Report: CSE-2005-2, 2005.

[4] J.Roberto Evaristo, Kevin C. Desouza, Kevin Hollister, "Centralization momentum: the pendulum swings back again", Communications of the ACM, Vol. 48 , Issue 2, February 2005, pp. 66-71.

[5] A. Neto et al., "Scalable Resource Provisioning for Multi-User Communications in Next Generation Networks", Global Telecommunications Conference, IEEE GLOBECOM 2008.

[6] Xiaomei Liu, Li Xiao, "A Survey of Multihoming Technology in Stub Networks: Current Research and Open Issues", MNET.2007.364256.

[7] Jun Bi, Ping $\mathrm{Hu}$, Lizhong Xie, "Site Multihoming: Practices, Mechanisms and Perspective", Future generation communication and networking, FGCN.2007.202.

[8] S.H.L. Liang, "A New Fully Decentralized Scalable Peer-to-Peer GIS Architecture", ISPRS XXIth Congress, Beijing 2008.

[9] T. Clausen, P. Jacquet; "Optimized Link State Routing Protocol (OLSR)", RFC 3626, October 2003.

[10] Guojun Jin, Brian L. Tierney, "System Capability Effects on Algorithms for Network Bandwidth Measurement", SIGCOMM 2003.

[11] Ningning $\mathrm{Hu}$, et al.; "locating Internet Bottlenecks: Algorithms, Measurements, and Implications", SIGCOMM'04.

[12] E. Cerqueira et al. "A Unifying Architecture for Publish-Subscribe Services in the Next Generation IP Networks". Published on IEEE GLOBECOM MMCOM'06, San Francisco, CA, US, Nov/Dec 2006.

[13] Yuri Breitbart et al., "Efficient monitoring bandwidth and latency in IP networks", Proceeding of IEEE INFOCOM, 2001.

[14] Susana Sargento, Rui Valadas; "Accurate estimation of capacities and cross-traffic of all links in a path using ICMP timestamps", Telecommunication Systems Journal, Vol. 33, No. 1-3, pp. 89-115, Dec 2006.

[15] Salehin, K.M.; Rojas-Cessa, R.; "Combined methodology for measurement of available bandwidth and link capacity in wired packet networks", Communications Journal, IET, Volume 4, Issue 2, January 222010 Page(s):240 - 252.

[16] K. Rose, S.L Regunathan; "Toward Optimality in Scalable Predictive Coding", VOL. 10, NO. 7, JULY 2001. 